\title{
Evaluación del diseño de un programa de intervención gamificado para el desarrollo del pensamiento histórico en el alumnado de Educación Primaria ${ }^{1}$
}

\author{
María MARTÍNEZ-HITA \\ Pedro MIRALLES-MARTÍNEZ
}

Datos de contacto:

María Martínez-Hita

Universidad de Murcia

m.martinezhita@um.es

Pedro Miralles-Martínez

Universidad de Murcia

pedromir@um.es

\section{RESUMEN}

En las últimas décadas, el desarrollo del pensamiento histórico en el alumnado ha adquirido una mayor importancia en la educación histórica, así como la necesidad de modificar y mejorar los métodos y estrategias de enseñanza. Debido a esto, se ha diseñado un programa educativo basado en la gamificación, teniendo en consideración la base teórica al respecto. El programa ha sido diseñado para aportar una alternativa a las usuales prácticas de enseñanza que se llevan a cabo en muchas clases de Educación Primaria, especialmente cuando se enseñan los contenidos históricos. El objetivo de este artículo es evaluar y validar el diseño del programa de intervención gamificado para desarrollar el pensamiento histórico en el alumnado de cuarto de Educación Primaria. La validación fue llevada a cabo por un panel de cuatro expertos con experiencia en el tema, Didáctica de la Historia y las Ciencias Sociales, que completaron un cuestionario ya validado, con una escala Likert de cinco puntos. Esta investigación es de carácter cuantitativo descriptivo y los resultados muestran que, aunque es necesario introducir algunas modificaciones para mejorar ciertas áreas del programa, el diseño es válido y altamente apropiado. Por tanto, el programa educativo gamificado puede ser implementado en el aula tras realizar los cambios necesarios.

PALABRAS CLAVE: enseñanza de la historia; innovación pedagógica; enseñanza primaria; programas de educación; investigación didáctica.

1 Este trabajo es resultado del proyecto de investigación "El pensamiento geográfico e histórico del alumnado de Educación Primaria en la Región de Murcia: propuesta metodológica innovadora para una educación de calidad" (20874/Pl/18). Proyecto financiado por la Comunidad Autónoma de la Región de Murcia a través de la convocatoria de Ayudas a proyectos para el desarrollo de investigación científica y técnica por grupos competitivos, incluida en el Programa Regional de Fomento de la Investigación Científica y Técnica (Plan de Actuación 2018) de la Fundación Séneca-Agencia de Ciencia y Tecnología de la Región de Murcia. 


\title{
Evaluation of the design of a gamified intervention program to develop historical thinking competencies in primary school students
}

\begin{abstract}
Over the last decades, the importance of developing students' historical thinking skills has gained more attention in history education, as well as the necessity to change and improve teaching methods and strategies. Because of this, an educational program based on gamification has been designed considering the theoretical basis in this regard. The program has been designed to provide an alternative to the teaching practices common in many elementary classrooms, especially when the historical contents are taught. The aim of this research paper is to evaluate and validate the design of the gamified educational programme to develop historical thinking competencies in primary school students. The validation process was conducted by a panel of four experts experienced in the subject matter, Didactics of History and Social Sciences, completing a validated questionnaire with a 5-point Likert scale. It is a quantitative descriptive research and the results show that, although it is necessary to introduce some modifications to improve a few areas of the programme, the design is valid and highly appropriate. Therefore, the gamified educational programme can be implemented at school after making the necessary changes.
\end{abstract}

KEYWORDS: History education; Teaching method innovations; Primary education; Educational programmes; Educational research.

\section{Introducción}

\section{La enseñanza de la historia}

Las investigaciones sobre la enseñanza de la historia coinciden en la necesidad de que su aprendizaje no se base únicamente en la memorización de datos, fechas o conceptos, sino que incluya habilidades y competencias que posibiliten el análisis, interpretación y explicación del pasado (Dominguez, 2015; Lee, 2005; Lee \& Ashby, 2000; Prats \& Santacana, 2011; VanSledright, 2011; Wineburg, 2001).

Por tanto, se defiende que hay que enseñar a pensar históricamente, diferenciando dos tipos de conceptos históricos complementarios e imprescindibles. Un conocimiento sustantivo de primer orden sobre el pasado que son conocimientos concretos, datos, 
hechos, fechas y personajes del pasado. Y un conocimiento histórico de segundo orden que aporta las herramientas necesarias para el estudio del pasado y la construcción de explicaciones históricas (Lee, 2005; Lee \& Ashby, 2000).

En las últimas décadas, el desarrollo del pensamiento histórico en el alumnado ha adquirido una mayor importancia. Se busca que los estudiantes avancen desde un papel pasivo, en el que asumen el pasado como fijo e invariable, a un conocimiento más profundo de la disciplina, conociendo los métodos y procedimientos de la investigación histórica (Gómez \& Miralles, 2017; Peck \& Seixas, 2008; Stoel, Logtenberg, Wansink, Huijgen, van Boxtel \& van Drie, 2017). Además, de esta forma, se contribuye a acabar con las concepciones del alumnado sobre los conocimientos históricos como poco útiles y aburridos (Burenheide, 2007; Liceras, 2016; Llopis \& Balaguer, 2016; Sanz, Molero \& Rodríguez, 2017; Van Straaten, Wilschut \& Oostdam, 2018).

La importancia del pensamiento histórico se refleja en los currículos de muchos países como Reino Unido (DfE, 2014), Canadá (Ontario Ministry of Education, 2018), Australia (ACARA, 2015), México (SEP, 2017) o Chile (Ministerio de Educación, 2012), entre otros.

En España también se hace necesaria una redefinición del modelo de educación histórica del alumnado, entre otras razones, por la casi nula introducción del método del historiador en el aula (Gómez \& Miralles, 2017) y la ausencia de los conceptos de segundo orden tanto en el currículo (Martínez-Hita, 2019; Martínez-Hita \& Gómez, 2016) como en los libros de texto (Martínez-Hita \& Gómez, 2018).

Asimismo, la necesidad de introducir otros métodos de enseñanza que permitan al alumnado adquirir progresivamente las competencias de pensamiento histórico se justifica por las finalidades y epistemología de la historia, así como por las orientaciones que se recogen en los propios currículos educativos (Gómez, Ortuño \& Miralles, 2018).

Partiendo de esta necesidad, en esta investigación se ha diseñado un programa de intervención, destinado al alumnado de Educación Primaria en España, que pretende que el trabajo de las competencias de pensamiento histórico se apoye en metodologías activas de aprendizaje como en la gamificación, que está alcanzando una gran popularidad en los últimos años (Hamari, Koivisto \& Sarsa, 2014; Kocakoyun \& Ozdamli, 2018). 


\section{La gamificación como estrategia}

La gamificación consiste en hacer uso de elementos que suelen formar parte de los juegos y aplicarlos en la educación para mejorar la experiencia de aprendizaje, consiguiendo estimular la atención y motivación del alumnado (Özdener, 2018; Robson, Plangger, Kietzmann, Mccarthy \& Pitt, 2015). De una forma más sencilla, se podría decir que la gamificación consiste en introducir las dinámicas y mecánicas propias del juego en el aula (Rivero, 2017).

La gamificación, atendiendo a Werbach \& Hunter (2012), se sustenta en tres elementos principales: dinámicas, mecánicas y componentes.

Las dinámicas son los aspectos más generales de la gamificación como las limitaciones, emociones, narrativa o progresión. Las mecánicas son los componentes básicos que estimulan la participación y el desarrollo de la actividad como los retos, recompensas, competición, cooperación o feedback. Los componentes son los elementos específicos y concretos del juego como avatares, insignias, puntos, niveles, tablas de clasificación, etc.

No obstante, esto no significa que las clases se conviertan en un juego, ya que la gamificación tiene un objetivo concreto que es el aprendizaje, pero desde un punto de vista motivador (Kapp, 2012). De este modo, es posible trabajar las competencias de pensamiento histórico y al mismo tiempo tratar de acabar con la desmotivación del alumnado al considerar la historia aburrida (Llopis \& Balaguer, 2016; Sanz et al., 2017), pues la gamificación fomenta la participación y la implicación del alumnado gracias al estímulo de conseguir recompensas y lograr el reto o misión propuesta (Rivero, 2017).

La mayoria de las investigaciones previas realizadas muestran los beneficios de introducir la gamificación en el aula, que se refleja en el aprendizaje, la motivación o participación del alumnado, entre otros aspectos (Chapman \& Rich, 2018; Da Rocha Seixas, Gomes, \& De Melo Filho, 2016; Hamari et al., 2014; Majuri, Koivisto \& Hamari, 2018; Ortiz-Colón, Jordán \& Agredal, 2018; Özdener, 2018; Yildirim, 2017).

Además, la gamificación, junto con otros métodos activos de aprendizaje, permite una participación activa del alumnado en la construcción de su propio aprendizaje como defiende la teoría constructivista cuyos principios son coherentes con la investigación empírica en el campo de la educación histórica (Van Straaten et al., 2018).

Sin embargo, la mayoría de las experiencias gamificadas son llevadas a cabo en niveles superiores como Educación Secundaria, 
Bachillerato y Educación superior (Kocakoyun \& Ozdamli, 2018; OrtizColón et al., 2018), existiendo una laguna de investigación en la Educación Primaria. Por tanto, se muestra de nuevo la necesidad de diseñar un programa de intervención gamificado para desarrollar el pensamiento histórico en este nivel educativo.

\section{Objetivos}

El diseño e implementación de un programa de intervención tiene que ir acompañado de una investigación evaluativa. La evaluación de los programas educativos es un aspecto fundamental que nos permite obtener información rigurosa para realizar juicios de valor con el fin de tomar decisiones de mejora y de cambio (Pérez, 2004).

Pérez Juste (2000), en su propuesta evaluativa de programas educativos, destaca la relevancia de llevar a cabo una primera evaluación del programa en su conjunto que esté orientada a la mejora del mismo antes de que sea implementado.

Por consiguiente, el principal objetivo de este artículo es evaluar y validar el diseño del programa gamificado elaborado para el desarrollo del pensamiento histórico en el alumnado de $4 .^{\circ} \mathrm{de}$ Educación Primaria antes de su implementación en el aula.

\section{Método}

\section{Enfoque}

Esta investigación es de carácter cuantitativo descriptivo (Latorre, Del Rincón \& Arnal, 2003), ya que presenta los estadísticos obtenidos tras la evaluación del diseño del programa de intervención gamificado con el objetivo de validarlo.

\section{Programa formativo}

El programa de intervención diseñado consta de una serie de retos y misiones que el alumnado debe superar para conseguir puntos e insignias que le permitirán alcanzar un determinado nivel según su desempeño. Se trata de un proyecto global e inclusivo que pretende dar respuesta a la diversidad mediante actividades que todo el alumnado es capaz de realizar dentro de sus posibilidades, haciendo uso de metodologias inclusivas y activas (Guillén, 2018; Lata \& Castro, 2016).

El programa pretende trabajar de manera integrada las 
competencias del pensamiento histórico junto con los elementos prescriptivos del currículo escolar (CARM, Decreto 198/2014). Está destinado al alumnado de $4 .^{\circ}$ de Educación Primaria y será puesto en práctica en la asignatura de Ciencias Sociales.

El hilo conductor que permite aunar todas las misiones del proyecto es la narrativa, aspecto básico de la gamificación. La historia, que es la linea narrativa de estas misiones, es la siguiente:

Somos una misión de extraterrestres que ha estado en la Tierra durante todo el curso investigando sobre los humanos y cumpliendo con las misiones que nos ha enviado nuestra Jefa Superiora. Estamos divididos en cinco grupos de trabajo, cada grupo procede de un planeta diferente, pero ha llegado el momento de volver cada uno a su planeta. Sin embargo, existe un problema, la Jefa Superiora quiere comprobar si realmente nos merecemos volver a nuestro planeta. Nuestras naves espaciales están bloqueadas y no conocemos las claves de acceso para que funcionen de nuevo. Para conseguir estos números que nos permitan volver debemos completar una serie de misiones. Además, según nuestro trabajo en las misiones conseguiremos puntos que determinarán cómo seremos recordados o consideramos en nuestro planeta cuando regresemos. Depende de nosotras y nosotros.

Además, para una mayor inmersión del alumnado en la gamificación, cada discente tendrá un avatar que simula ser un extraterrestre, usando para ello el programa ClassDojo que permite igualmente la asignación de puntos.

El programa de intervención queda resumido en la Figura 1 que es un esquema de las misiones que componen el proyecto y será entregado al alumnado en la primera sesión para que le sirva como guía. 


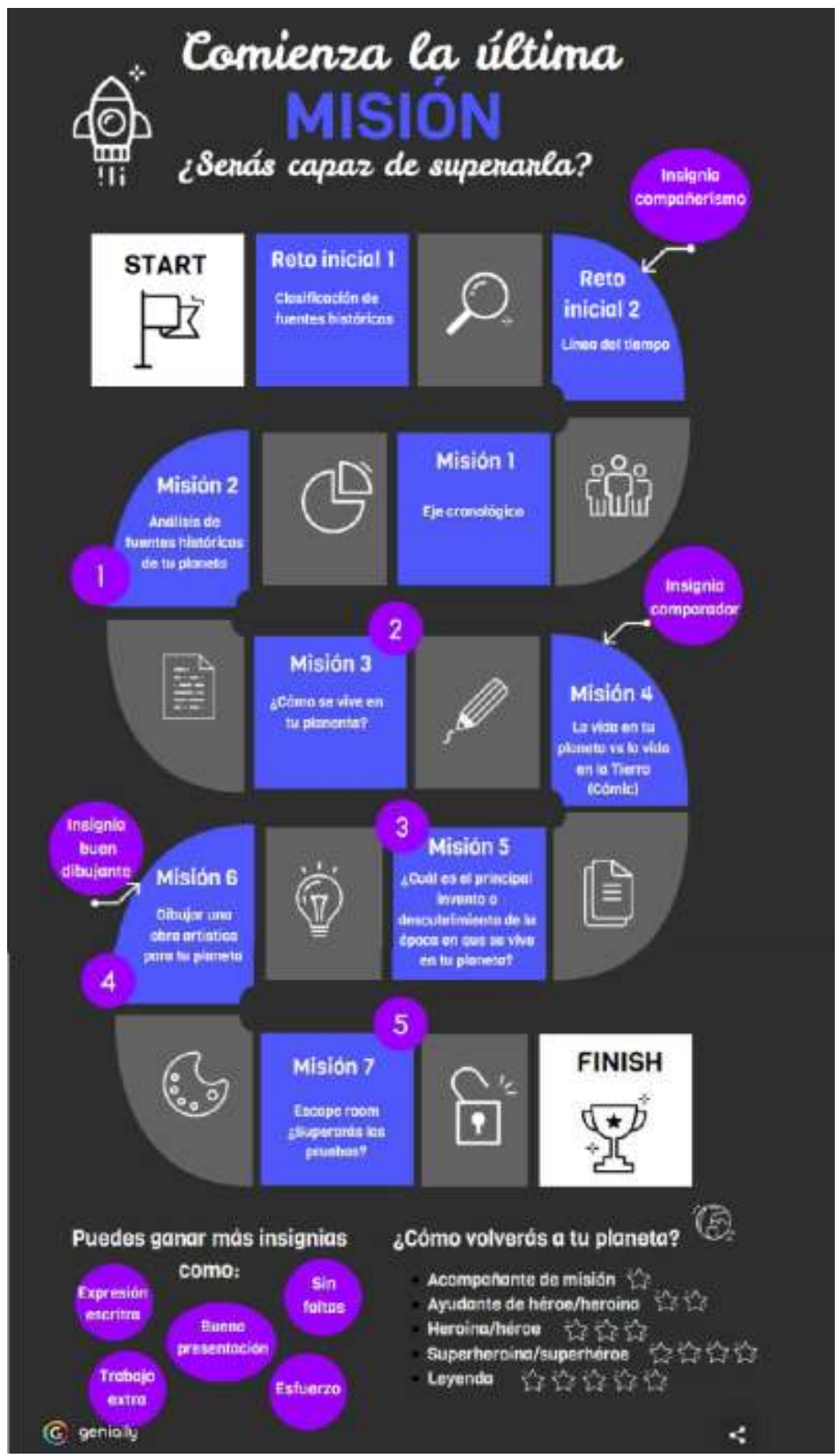

Figura 1. Esquema de las misiones del proyecto de intervención gamificado.

Fuente: elaboración propia 
En esta Figura 1 se observan igualmente otros elementos de la gamificación como las insignias o los niveles que el alumnado puede alcanzar al finalizar las misiones según su progreso y logros.

\section{Instrumento de análisis}

Para la recogida de la información se ha utilizado una adaptación del instrumento ${ }^{1}$ diseñado por Maquilón (2003).

El instrumento de evaluación se trata de una lista de control autoaplicada, que permite la validación del programa diseñado. Consta de ocho fichas que integran los indicadores correspondientes a ocho criterios preestablecidos, incluyendo en su conjunto 39 ítems. Cada indicador se acompaña de una escala Likert cuyos valores oscilan entre uno (nunca) y cinco (siempre).

Cada una de estas ocho fichas, que hace referencia a un criterio diferente atendiendo a las diversas publicaciones realizadas por el profesor Pérez Juste sobre esta temática, se agrupan en cuatro dimensiones como muestra la Tabla 1.

Tabla 1

Dimensiones y criterios evaluados en el instrumento de análisis

\begin{tabular}{|c|c|c|}
\hline DIMENSIÓN & CRITERIO & DESCRIPCIÓN \\
\hline \multirow{3}{*}{ ENDÓGENA } & Contextualizado & $\begin{array}{l}\text { Adecuada a las características y necesidades del } \\
\text { contexto para el que ha sido diseñado }\end{array}$ \\
\hline & Consistente & $\begin{array}{l}\text { Potente como UD en sí, con la suficiente calidad } \\
\text { técnica y los elementos que deben configurar } \\
\text { una UD }\end{array}$ \\
\hline & Congruente & $\begin{array}{l}\text { Basada en un modelo teórico de contrastada } \\
\text { validez, además de coherente a nivel pedagógico } \\
\text { y en su desarrollo. }\end{array}$ \\
\hline \multirow{2}{*}{$\begin{array}{l}\text { ENDÓGENA- } \\
\text { EXÓGENA }\end{array}$} & Flexible & $\begin{array}{l}\text { Adaptable, dinámica, abierta, que puede } \\
\text { adecuarse a situaciones diversas }\end{array}$ \\
\hline & Conveniente & $\begin{array}{l}\text { Util para dar respuesta a las necesidades } \\
\text { detectadas, eficaz en el logro de los objetivos. }\end{array}$ \\
\hline EXÓGENA & Factible & $\begin{array}{l}\text { Aceptable desde el punto de vista de la } \\
\text { institución responsable y, ejecutable si existe la } \\
\text { disponibilidad de espacios y recursos materiales }\end{array}$ \\
\hline
\end{tabular}

1 La adaptación ha sido elaborada en el marco del proyecto de investigación "El pensamiento geográfico e histórico del alumnado de Educación Primaria en la Región de Murcia: propuesta metodológica innovadora para una educación de calidad" (20874/PI/18) coordinado por Pedro Miralles. 
Evaluación del diseño de un programa de intervención gamificado para el desarrollo del pensamiento histórico en el

\begin{tabular}{lll}
\hline DIMENSIÓN & CRITERIO & DESCRIPCIÓN \\
\cline { 2 - 4 } & Generalizable & $\begin{array}{l}\text { Aplicable a situaciones diversas dentro del } \\
\text { mismo contexto, y en otros contextos de } \\
\text { caracteristicas similares }\end{array}$ \\
\hline Evaluable & $\begin{array}{l}\text { Permite realizar juicios de valor y toma de } \\
\text { decisiones sobre la UD y todos los aspectos } \\
\text { relacionados a su aplicación, resultados y la } \\
\text { propia evaluación. }\end{array}$ \\
\hline
\end{tabular}

Fuente: elaboración propia

\section{Procedimiento y análisis de datos}

Una vez diseñado el programa de intervención para el trabajo de las competencias de pensamiento histórico, cuatro evaluadores externos, expertos en la didáctica de las ciencias sociales, evaluaron la propuesta didáctica a través del instrumento anteriormente mencionado.

El programa para llevar a cabo el análisis estadístico ha sido SPSS v.19. Los datos obtenidos fueron introducidos en dicho programa para su análisis descriptivo.

El criterio que se siguió para interpretar los resultados fue considerar como aceptables las puntuaciones de la mediana mayores o iguales a 3.5, lo que indicará que la mitad de los evaluadores se sitúan por encima de ese valor.

Asimismo, para determinar el grado de concordancia entre los evaluadores se ha realizado una prueba no paramétrica de comparación de $\mathrm{K}$ medias dependientes o relacionadas, la $\mathrm{W}$ de Kendall.

\section{Resultados}

Los resultados obtenidos por parte de los evaluadores se presentan diferenciando cada una de las dimensiones en las que se divide el instrumento de evaluación del programa.

En la Tabla 2 aparecen los estadísticos de los criterios contextualizado, consistente y congruente que conforman la dimensión endógena. Todos los ítems se sitúan por encima del valor considerado como aceptable, excepto el ítem número 22 que su mediana es exactamente el valor 3.5.

Tabla 2

Estadísticos de la dimensión endógena 


\begin{tabular}{|c|c|c|c|c|c|c|c|}
\hline Criterio & & $\mathrm{N}$ & Media & Mediana & $\begin{array}{c}\text { Desviación } \\
\text { estándar }\end{array}$ & Mínimo & Máximo \\
\hline Contextua- & Ítem_1 & 4 & 4,00 & 4,00 & 1,155 & 3 & 5 \\
\hline lizado & Ítem_2 & 4 & 4,50 & 5,00 & 1,000 & 3 & 5 \\
\hline \multirow[t]{11}{*}{ Consistente } & Ítem_3 & 4 & 4,75 & 5,00 &, 500 & 4 & 5 \\
\hline & Ítem_4 & 4 & 4,75 & 5,00 &, 500 & 4 & 5 \\
\hline & Ítem_5 & 4 & 4,75 & 5,00 &, 500 & 4 & 5 \\
\hline & Ítem_6 & 4 & 4,50 & 5,00 & 1,000 & 3 & 5 \\
\hline & Ítem_7 & 4 & 4,25 & 4,50 & ,957 & 3 & 5 \\
\hline & Ítem_8 & 4 & 5,00 & 5,00 & ,000 & 5 & 5 \\
\hline & Ítem_9 & 4 & 5,00 & 5,00 & ,000 & 5 & 5 \\
\hline & Ítem_10 & 4 & 5,00 & 5,00 & ,000 & 5 & 5 \\
\hline & Ítem_11 & 4 & 5,00 & 5,00 &, 000 & 5 & 5 \\
\hline & Ítem_12 & 4 & 4,50 & 5,00 & 1,000 & 3 & 5 \\
\hline & Ítem_13 & 4 & 4,50 & 5,00 & 1,000 & 3 & 5 \\
\hline \multirow[t]{11}{*}{ Congruente } & Ítem_14 & 4 & 5,00 & 5,00 & ,000 & 5 & 5 \\
\hline & Ítem_15 & 4 & 4,50 & 5,00 & 1,000 & 3 & 5 \\
\hline & Ítem_16 & 4 & 4,50 & 5,00 & 1,000 & 3 & 5 \\
\hline & Ítem_17 & 4 & 4,50 & 5,00 & 1,000 & 3 & 5 \\
\hline & Ítem_18 & 4 & 5,00 & 5,00 & ,000 & 5 & 5 \\
\hline & Ítem_19 & 4 & 5,00 & 5,00 & ,000 & 5 & 5 \\
\hline & Ítem_20 & 4 & 4,75 & 5,00 &, 500 & 4 & 5 \\
\hline & Ítem_21 & 4 & 4,50 & 4,50 &, 577 & 4 & 5 \\
\hline & Ítem_22 & 4 & 3,25 & 3,50 & 2,062 & 1 & 5 \\
\hline & Ítem_23 & 4 & 4,50 & 5,00 & 1,000 & 3 & 5 \\
\hline & Ítem_24 & 4 & 4,50 & 5,00 & 1,000 & 3 & 5 \\
\hline
\end{tabular}

Fuente: elaboración propia

La Tabla 3 muestra los estadísticos de los criterios flexible y conveniente que conforman la dimensión endógena-exógena. Todos los items tienen una puntuación mínima de tres, esto es, por encima del valor medio de la escala. 


\section{Tabla 3}

Estadísticos de la dimensión endógena-exógena

\begin{tabular}{lccrrrrr}
\hline Criterio & & N & Media & Mediana & $\begin{array}{c}\text { Desviación } \\
\text { estándar }\end{array}$ & Mínimo & Máximo \\
\hline \multirow{2}{*}{ Flexible } & Ítem_25 & 4 & 4,50 & 4,50 &, 577 & 4 & 5 \\
& Ítem_26 & 4 & 4,50 & 4,50 &, 577 & 4 & 5 \\
& Ítem_27 & 4 & 4,50 & 4,50 &, 577 & 4 & 5 \\
& Ítem_28 & 4 & 4,50 & 4,50 &, 577 & 4 & 5 \\
& Ítem_29 & 4 & 4,25 & 4,50 &, 957 & 3 & 5 \\
\hline \multirow{2}{*}{ Conveniente } & Ítem_30 & 4 & 5,00 & 5,00 &, 000 & 5 & 5 \\
& Ítem_31 & 4 & 4,50 & 5,00 & 1,000 & 3 & 5 \\
\hline
\end{tabular}

Fuente: elaboración propia

Del mismo modo, la Tabla 4 muestra que los tres items de la dimensión exógena referidos a los criterios factible y generalizable presentan unas valoraciones altas.

Tabla 4

Estadísticos de la dimensión exógena

\begin{tabular}{lrrrrrrr}
\hline Criterio & & N & Media & Mediana & $\begin{array}{c}\text { Desviación } \\
\text { estándar }\end{array}$ & Mínimo & Máximo \\
& & & & & & \\
Factible & Ítem_32 & 4 & 4,50 & 5,00 & 1,000 & 3 & 5 \\
& Ítem_33 & 4 & 4,50 & 5,00 & 1,000 & 3 & 5 \\
\hline Generalizable & Ítem_34 & 4 & 4,75 & 5,00 &, 500 & 4 & 5 \\
\hline
\end{tabular}

Fuente: elaboración propia

El último criterio, perteneciente a la dimensión integral, valora en qué medida el programa es evaluable. Todos los items superan ampliamente el valor establecido como aceptable, excepto el item 39 que obtiene una puntuación exactamente a dicho valor (véase Tabla 5).

Tabla 5

Estadisticos de la dimensión integral

\begin{tabular}{lrrrrrrr}
\hline Criterio & & N & Media & Mediana & $\begin{array}{c}\text { Desviación } \\
\text { estándar }\end{array}$ & Mínimo & Máximo \\
& & & & & \\
\hline \multirow{2}{*}{ Evaluable } & Ítem_35 & 4 & 4,50 & 4,50 &, 577 & 4 & 5 \\
& Ítem_36 & 4 & 4,50 & 4,50 &, 577 & 4 & 5
\end{tabular}




\begin{tabular}{rrrrrrr}
\hline Ítem_37 & 4 & 4,50 & 5,00 & 1,000 & 3 & 5 \\
Ítem_38 & 4 & 5,00 & 5,00 &, 000 & 5 & 5 \\
Ítem_39 & 4 & 3,25 & 3,50 & 2,062 & 1 & 5 \\
\hline
\end{tabular}

Fuente: elaboración propia

Por último, en la Tabla 6 se observa el nivel de concordancia de las valoraciones realizadas por los evaluadores externos sobre el programa de intervención. Los resultados indican que existe concordancia entre ellos $(\mathrm{p}<.05)$, siendo el grado de la misma medio $(\mathrm{W}=.395)$.

\section{Tabla 6}

Estadisticos de contraste

\begin{tabular}{ll}
\hline $\mathrm{N}$ & 39 \\
W de Kendalla &, 395 \\
Chi-cuadrado & 46,269 \\
gl & 3 \\
Sig. asintót. &, 000 \\
\hline
\end{tabular}

a. Coeficiente de concordancia de

Kendall

Fuente: elaboración propia

\section{Discusión y conclusión}

Los resultados muestran que todas las valoraciones de los items realizadas por los expertos se sitúan por encima de la puntuación mínima establecida para considerar aceptable el diseño del programa de intervención. Además, la prueba no paramétrica de W de Kendall indica que existe concordancia entre los evaluadores.

No obstante, aunque prácticamente todos los items tienen tanto una media como mediana superior a cuatro, los items 22 y 39 no superan esta puntuación y, además, cuentan con una desviación estándar elevada. Esto refleja cierta disparidad en las evaluaciones de los expertos de estos dos ítems y la necesidad de hacer modificaciones que contribuyan a la mejora de estos dos aspectos.

El item 22 se refiere a si "la UD tiene en cuenta la atención a la diversidad del alumnado a través de medidas ordinarias y específicas".

El proyecto diseñado busca ofrecer una educación común para las necesidades y posibilidades de cada discente en vez de adaptaciones curriculares individuales, ya que, tras el análisis del 
contexto, el alumnado no precisaba de dichas adaptaciones específicas.

Como indican Rojas y Haya (2018): "Cuanto más adaptada esté la programación del aula a la heterogeneidad del grupo, menos necesarias son las adaptaciones que se realizan individualmente con algunos estudiantes" (p. 149).

La puntuación obtenida en este ítem tiene su explicación en que en la secuencia de enseñanza-aprendizaje presentada a los expertos no se especificaban medidas de atención a la diversidad, ya que dicha diversidad ya fue tomada en consideración cuando se diseñó el proyecto.

Se ha pretendido diseñar actividades amplias, con distintos niveles de dificultad, de forma que el alumnado en función de sus capacidades pueda llevarlas a cabo ya sea simplificando o haciéndolas más complejas. A esto hay que sumar el uso de metodologías activas y el trabajo cooperativo que permiten la tutoría entre iguales y la ayuda mutua dentro de equipos pequeños y heterogéneos, lo cual favorece la inclusión educativa (Guillén, 2018; Lata \& Castro, 2016).

Además, para mejorar la atención a la diversidad, el nivel de apoyo por parte del docente será mayor en aquel alumnado con necesidades, ya que la metodología de trabajo permite una ayuda más individualizada. Asimismo, el tiempo de ejecución de las tareas será flexible, reconociendo el tiempo adicional que necesitan algunos discentes. Se facilitarán igualmente recursos de apoyo, usando materiales diversos y diferentes vías de información.

Por su parte, el ítem 39 hace alusión a si "el proceso de evaluación culmina con una evaluación de la evaluación (metaevaluación)".

El proyecto finaliza con una última sesión en la que se establece un coloquio entre los discentes y el docente en el que se reflexionará sobre todo el proyecto. Tras las valoraciones del juicio de expertos, se incluirá una autorreflexión de los estudiantes para obtener su opinión sobre los resultados obtenidos en la evaluación y, de esta forma, conseguir una evaluación de la evaluación. Todo esto planteado de tal forma que siga dentro de la narrativa de la gamificación planteada.

El docente también realizará una autorreflexión crítica sobre las prácticas evaluativas (Sime, 1998), instrumentos de evaluación y todo el proyecto en su conjunto para determinar su adecuación y pertinencia. Por consiguiente, cuando finalice el proceso, se reflexionará sobre la práctica que incluye la práctica evaluadora. El maestro revisará el modo de ejecutar la evaluación, sometiendo a evaluación el propio proceso evaluador (Castillo \& Cabrerizo, 2010).

Por tanto, de acuerdo con los datos obtenidos en la valoración 
del resto de ítems, así como con los cambios que se van a introducir con la finalidad de mejorar los aspectos que han obtenido peores puntuaciones, es posible validar el programa de intervención diseñado. Esta primera evaluación del programa en su conjunto, que ha permitido determinar su calidad técnica, viabilidad práctica y "evaluabilidad" (Pérez Juste, 2000), asegura las condiciones óptimas del mismo para su implementación en el aula.

\section{Referencias}

ACARA (Australian Curriculum Assessment and Reporting Authority (2015). The Australian Curriculum: Humanities and Social Sciences. History. Version 8.1. Sídney: ACARA. Recuperado de http://www.australiancurriculum.edu.au/

Ayén, F. (2017). ¿Qué es la gamificación y el ABJ? Íber. Didáctica de las Ciencias Sociales, Geografía e Historia, 86, 7-15.

Burenheide, B. (2007). I Can Do This: Revelations on Teaching with Historical Thinking. The History Teacher, 41(1), 55-61. DOI: $10.2307 / 30037103$

Castillo, S., \& Cabrerizo, J. (2010). Evaluación Educativa de aprendizajes y competencias. Madrid: Pearson Educación.

Chapman, J. R., \& Rich, P. J. (2018). Does educational gamification improve students' motivation? If so, which game elements work best? Journal of Education for Business, 93(7), 315-322. DOI: 10.1080/08832323.2018.1490687

Comunidad Autónoma de la Región de Murcia (2014). Decreto $198 / 2014$, de 5 de septiembre, por el que se establece el currículo de la Educación Primaria en la Comunidad Autónoma de la Región de Murcia. Boletín Oficial de la Región de Murcia, 206, 6 de septiembre de 2014.

Da Rocha Seixas, L., Gomes, A. S., \& De Melo Filho, I. J. (2016). Effectiveness of gamification in the engagement of students. Computers in Human Behavior, 58, 48-63. DOI: http://dx.doi.org/10.1016/j.chb.2015.11.021

DfE (2014). The National Curriculum in England: Framework document. London: DfE. Recuperado de https://www.gov.uk/

Domínguez, J. (2015). Evaluación del pensamiento histórico y 
conceptos metodológicos de la historia. En Pensamiento histórico y evaluación de competencias (pp. 35-64). Barcelona: Graó.

Gibson, D., Ostashewski, N., Flintoff, K., Grant, S., \& Knight, E. (2015). Digital badges in education. Education and Information Technologies, 2O(2), 403-410. DOI: 10.1007/s10639-0139291-7

Gómez, C. J. \& Miralles, P. (2017). Los espejos de Clío. Usos y abusos de la Historia en el ámbito escolar. Madrid: Sílex.

Gómez, C. J., Ortuño, J., \& Miralles, P. (2018). Enseñar ciencias sociales con métodos activos de aprendizaje. Reflexiones y propuestas a través de la indagación. Barcelona: Octaedro

Guillén, J. (coord.) (2018). Guía metodológica sobre dificultades específicas de aprendizaje. Murcia: Consejería de Educación, Juventud y Deportes. Secretaria General. Servicio de Publicaciones y Estadística.

Hamari, J., Koivisto, J., \& Sarsa, H. (2014). Does Gamification Work? - A Literature Review of Empirical Studies on Gamification. En 2014 47th Hawaii International Conference on System Science (HICSS) (pp. 3025-3034). DOI: 10.1109/HICSS.2014.377

Kapp, K. M. (2012). The Gamification of Learning and Instruction. Game-Based Methods and Strategies for Training and Education. San Francisco: John Wiley \& Sons.

Kocakoyun, S., \& Ozdamli, F. (2018). A Review of Research on Gamification Approach in Education. En R. Morese (ed.), Socialization. A Multidimensional Perspective (pp. 51-73). London: IntechOpen.

DOI: http: / /dx.doi.org/10.5772/intechopen.74131

Lata, S., \& Castro, M. M. (2016). El Aprendizaje Cooperativo, un camino hacia la inclusión educativa. Revista Complutense de Educación, 27(3), 1085-1101.

DOI: http://dx.doi.org/10.5209/rev_RCED.2016.v27.n3.47441

Latorre, A., del Rincón, D., \& Arnal, J. (2003). Investigación evaluativa. En A. Latorre, D. del Rincón \& J. Arnal, Bases metodológicas de la investigación educativa (pp. 243-272). Barcelona: Ediciones Experiencia. 
Lee, P., \& Ashby, R. (2000). Progression in Historical Understanding among Students Ages 7-14. En P. N. Stearns, P. Seixas \& S. Wineburg (eds.), Knowing, Teaching, and Learning History: National and International Perspectives (pp. 199-222). New York: New York University Press.

Lee, P. (2005). Putting Principles into Practice: Understanding History. En M. S. Donovan \& J. D. Bransford (eds.), How students learn: History in the classroom (pp. 29-78). Washington, DC: National Academies Press.

Liceras, A. (2016). Las dificultades en la enseñanza y el aprendizaje de las ciencias sociales. En A. Liceras \& G. Romero (coords.), Didáctica de las Ciencias Sociales. Fundamentos, contextos y propuestas (pp. 95-118). Madrid: Pirámide.

Llopis, M. A., \& Balaguer, P. (2016). El uso del juego en educación. Gamificación. En O. Chiva \& M. Martí (coords.), Métodos pedagógicos activos y globalizadores (pp. 85-101). Barcelona: Graó.

Majuri, J., Koivisto, J., \& Hamari, J. (2018). Gamification of education and learning: A review of empirical literature. En Proceedings of the 2nd International GamiFIN Conference, Pori, Finland.

Maquilón, J. J. (2003). Diseño y Evaluación del Diseño de un Programa de Intervención para la Mejora de las Habilidades de Aprendizaje de los Estudiantes Universitarios (Tesis doctoral). Universidad de Murcia, Murcia.

Martínez-Hita, M (2019). Análisis comparativo del modelo cognitivo y conceptos históricos en el currículum español e inglés. En EIDUM (coord.), IV Jornadas Doctorales Escuela Internacional de Doctorado de la Universidad de Murcia (EIDUM) (pp. 560563). Murcia: Editum.

Martínez-Hita, M. \& Gómez, C. J. (2016). Diferentes enfoques sobre la enseñanza de la historia y del pensamiento histórico en el currículo español y canadiense. En J. Maquilón, C. J. Gómez \&M. B. Alfageme (eds.), De la investigación a la mejora educativa en las aulas (pp. 19-32). Murcia: Editum.

Martínez-Hita, M. \& Gómez, C. J. (2018). Nivel cognitivo y competencias de pensamiento histórico en los libros de texto de Historia de España e Inglaterra. Un estudio comparativo. 
Revista de Educación, 379, 145-169. DOI: 10.4438/1988592X-RE-2017-379-364.

Ministerio de Educación (2012). Bases curriculares. Historia, Geografia y Ciencias Sociales. Educación Básica. Santiago de Chile: Ministerio de Educación. Recuperado de www.curriculumenlineamineduc.cl

Ontario Ministry of Education (2018). The Ontario Curriculum: Social Studies, Grades 1 to 6; History and Geography, Grades 7 and 8. Ontario: Queen's Printer for Ontario. Recuperado de http://www.edu.gov.on.ca/eng/

Ortiz-Colón, A. M., Jordán, J., \& Agredal, M. (2018). Gamificación en educación: una panorámica sobre el estado de la cuestión. Educação e Pesquisa, 44, 1-17. DOI: http: / /dx.doi.org/10.1590/S1678-4634201844173773

Özdener, N. (2018). Gamification for enhancing Web 2.0 based educational activities: The case of pre-service grade school teachers using educational Wiki pages. Telematics and Informatics, 35, 564-578. DOI: http: / /dx.doi.org/10.1016/j.tele.2017.04.003

Peck, C., \& Seixas, P. (2008). Benchmarks of Historical Thinking: First Steps. Canadian Journal of Education, 31(4), 1015-1038.

Pérez Juste, R. (2000). La evaluación de programas educativos: conceptos básicos, planteamientos generales y problemática. Revista de Investigación Educativa, 18(2), 261-287.

Pérez, G. (2004). Modelos de investigación cualitativa en educación social y animación sociocultural: Aplicaciones prácticas. Madrid: Narcea Ediciones.

Prats, J., \& Santacana, J. (2011). Enseñar a pensar históricamente: la clase como simulación de la investigación histórica. En J. Prats (coord.), Didáctica de la Geografia y la Historia (pp. 67-89). Barcelona: Graó.

Rivero, P. (2017). Proceso de gamificación en el aula de ciencias sociales. Íber. Didáctica de las Ciencias Sociales, Geografia e Historia, 86, 4-6.

Robson, K., Plangger, K., Kietzmann, J. H., Mccarthy, I., \& Pitt, L. (2015). Is it all a game? Understanding the principles of 
gamification. Business Horizons, 58, 411-420. DOI: 10.1016/j.bushor.2015.03.006

Rojas, S., \& Haya, I. (2018). Fundamentos pedagógicos de atención a la diversidad. Cantabria: Universidad de Cantabria.

Sanz, P., Molero, J. M., \& Rodríguez, D. (eds.). (2017). La historia en el aula. Innovación docente y enseñanza de la historia en la educación secundaria. España: Milenio.

SEP (Secretaría de Educación Pública) (2017). Aprendizajes clave para la educación integral. Plan y programas de estudio para la educación básica. México: Secretaría de Educación Pública.

Sime, L. (1998). Metaevaluación: ir más allá de la evaluación para volver sobre ella. Educación, 7(14), 199-216.

Stoel, G., Logtenberg, A., Wansink, B., Huijgen, T., Van Boxtel, C., \& Van Drie, J. (2017). Measuring epistemological beliefs in history education: An exploration of naïve and nuanced beliefs. International Journal of Educational Research, 83, 120134. DOI: http://dx.doi.org/10.1016/j.ijer.2017.03.003

Van Straaten, D., Wilschut, A., \& Oostdam, R. (2018). Measuring students' appraisals of the relevance of history: The construction and validation of the Relevance of History Measurement Scale (RHMS). Studies in Educational Evaluation, 56, 102-111. DOI: https://doi.org/10.1016/j.stueduc.2017.12.002

VanSledright, B. A. (2011). The Challenge of Rethinking History Education. On Practice, Theories, and Policy. Nueva York: Routledge.

Werbach, K., \& Hunter D. (2012). For the Win: How Game Thinking Can Revolutionize Your Business. Philadelphia: Wharton Digital Press.

Wineburg, S. (2001). Historical Thinking and other Unnatural Acts. Charting the Future of Teaching the Past. Philadelphia: Temple University Press.

Yildirim, I. (2017). The effects of gamification-based teaching practices on student achievement and students' attitudes toward lessons. Internet and Higher Education, 33, 86-92. DOI: http://dx.doi.org/10.1016/j.iheduc.2017.02.002 